Trauma Berufskrankh 2017·19(Suppl3):S347-S349 DOI 10.1007/s10039-017-0286-2

Online publiziert: 3. August 2017

(c) Springer Medizin Verlag GmbH 2017

CrossMark

\section{B. Behr}

Klinik für Plastische Chirurgie und Schwerbrandverletzte, Handchirurgiezentrum, Operatives

Referenzzentrum für Gliedmaßentumoren, BG Universitätsklinikum Bergmannsheil, Bochum, Deutschland

\title{
Sekundäre Wiederherstellung von Nervenverletzungen und motorischer Ersatz an der oberen Extremität
}

\begin{abstract}
Auch wenn die Inzidenz von Nervenverletzungen im Rahmen von Traumata mit 1,6-2,8 \% relativ selten ist, können diese schwere Folgen nach sich ziehen. Dabei ist die Primärversorgung der Sekundärversorgung grundsätzlich vorzuziehen. Gelingt dies nicht, existieren verschiedene Strategien der sekundären Wiederherstellung von Nervenverletzungen sowie motorische Ersatzplastiken, um die Funktion des geschädigten Nervs zu rekonstruieren.
\end{abstract}

\section{Sekundäre Nerven- rekonstruktion}

Nervenverletzungen an den Extremitäten treten mit einer Inzidenz von etwa 1,6\% auf [9] und können lange Zeiten der Arbeitsunfähigkeit und hohe Kosten nach sich ziehen [7]. Im Falle eines Traumas sollte die Primärversorgung, d.h. innerhalb der ersten $72 \mathrm{~h}$, angestrebt werden [4]. Gelingt dies nicht, wird in der Regel zunächst die "delayed“ (innerhalb von 3 Wochen) oder sekundäre Nervenrekonstruktion favorisiert. Dabei ähneln die Grundprinzipien, spannungsfreie Koaptation und Anfrischen der Nervenenden, denen der Primärrekonstruktion. Sollte eine spannungsfreie Nervennaht nicht möglich sein, müssen Nerveninterponate, $\mathrm{z}$. B. vom N. suralis oder N. antebrachii medialis verwendet werden. Ein wichtiges Grundprinzip ist dabei, dass die Nerven im gesunden, nicht vernarbten Gewebe liegen, um die Regeneration zu ermöglichen. Im Anschluss an die Nervenrekonstruktion sind lange Wartezeiten von 6 Monaten und mehr notwendig, um das Endergebnis zu beurteilen. Neben der klassischen sekundären Nervenrekonstruktion mit Interponaten sind auch Nerventransfers und Neurotisationen als Techniken verfügbar. Bei der zuletzt genannten Technik kann durch eine End-zu-SeitNervennaht bei fehlenden proximalen Koaptationsmöglichkeiten eine proximale Nervenläsion in eine distale überführt werden. Hervorzuheben ist hier ebenfalls die Möglichkeit der sensiblen Reinnervation.

Die klinischen Ergebnisse der sekundären Nervenrekonstruktion an der oberen Extremität dürfen als durchwachsen bezeichnet werden. So kann man verallgemeinernd sagen, dass ein Drittel der Patienten ein gutes Ergebnis, ein Drittel ein befriedigendes Ergebnis und ein Drittel ein schlechtes Ergebnis erhalten. Zum Beispiel zeigte sich in einer großen Studie mit 260 Patienten zur Rekonstruktion des $\mathrm{N}$. radialis und $\mathrm{N}$. interosseus posterior, dass $30 \%$ der Patienten ein gutes Ergebnis hatten, allerdings bei $42 \%$ der Patienten die Heilung ausblieb. Sämtliche Rekonstruktionsversuche nach 12 Monaten scheiterten [8]. Zusammenfassend lässt sich sagen, dass sekundäre Nervenrekonstruktion im Falle des Erfolges physiologischen Prinzipien folgt und somit ein gutes Konzept darstellen kann. Allerdings sind die Erfolgsaussichten bei verzögerter Therapie und vernarbten oder bestrahlten Lokal- verhältnissen gering, sodass auf Alternativverfahren ausgewichen werden muss.

\section{Motorische Ersatzplastiken}

Eine Variante zur Wiederherstellung der Funktion an den Extremitäten stellen motorische Ersatzplastiken dar. Sie können sowohl an der oberen wie auch an der unteren Extremität durchgeführt werden und haben zum Prinzip, dass ein funktionierender Muskel mit Sehne auf eine Sehne ohne Funktion umgesetzt wird. Dabei sind zerebrale Lernvorgänge zur Reprogrammierung des Bewegungsmusters auch noch im hohen Alter möglich. Grundvoraussetzungen für eine motorische Ersatzplastik sind

- Gelenke, die passiv frei beweglich sind,

- kraftvolle Ersatzmuskeln, deren Funktion entbehrlich ist, und

- ein motivierter Patient, der gewillt ist, die anschließende Rehabilitation durchzuführen.

Weitere Prinzipien der motorischen Ersatzplastiken liegen in der direkten Sehnenführung, die möglichst ohne Umlenkung in einer geraden Linie erfolgen sollte, und der Auswahl eines Muskels mit der richtigen Bewegungsamplitude. Die Sehnen werden unter korrekter Vorspannung miteinander vernäht. In unserer Klinik wird meistens die Seit-zu-SeitKopplungstechnik nach Fridén verwen$\operatorname{det}$ [3]. 
An der oberen Extremität sind je nach Ausfall der Funktion verschiedene motorische Ersatzplastiken etabliert, von denen Ausgewählte im Folgenden vorgestellt werden. Eine häufig angewendete Ersatzplastik ist die Radialisersatzplastik bei Radialislähmung entweder durch direkte Schädigung des Nervs oder auch Kollateralschäden durch Frakturen am Humerus. Die Radialisersatzplastik kann in vielen verschiedenen Varianten durchgeführt werden, wobei sich bisher keine Kombination von Muskeln als überlegen durchgesetzt hat $[2,6,10]$. Allen Techniken gemeinsam ist, dass bei einem kompletten Radialisausfall 3 Funktionen wiederhergestellt werden müssen:

- die Hangelenkextension, ausgeführt durch die Extensor-carpi-radialisbrevis- und -longus-Sehnen,

- die Daumenextension durch die Extensor-pollicis-longus-Sehnen und

- die Fingerextension in den Metakarpophalangealgelenken durch die Extensor-digitorum-communisSehnen.

Je nach den individuellen Bedingungen des Patienten sowie den Präferenzen und der Erfahrung des Operateurs können dann verschiedene Techniken zum Einsatz kommen.

Die Ergebnisse nach der Radialisersatzplastik sind zufriedenstellend [1]. So konnte an einer Studie an der BG Klinik Ludwigshafen an 77 Patienten gezeigt werden, dass die Patienten nach Radialisersatzplastik eine akzeptable Funktion und wenig Schmerzen hatten. Besonders hervorzuheben ist es, dass $89 \%$ wieder in ihrem alten Beruf arbeiten konnten [1].

Die seltenere distale Ulnarisparese ist durch die Krallenstellung der Finger, Atrophie der Handbinnenmuskulatur und fehlende Adduktion des Daumens gekennzeichnet. Auch hier gibt es wieder verschiedene Methoden des motorischen Ersatzes. Zur Verbesserung der Flexion in den Metakarpophalangealgelenken wird gerne ein dynamischer Transfer, wie etwa eine aufgeteilte Sehne des Flexor digitorum superficialis IV, die durch die A2-Ringbänder der Langfinger geführt und dann mit sich selbst vernäht wird, verwendet [5]. Weitere
Eingriffe, wie beispielsweise der Transfer des Extensor carpi radialis brevis zum Adduktorenersatz, können je nach Patientenanforderungen erfolgen.

Eine weitere an der oberen Extremität häufig eingesetzte motorische Ersatzplastik ist der Extensor-indicis-Transfer bei isoliertem Ausfall der Extensor-pollicis-longus-Sehne. Diese Verletzung ist meistens traumatischer oder degenerativer Genese und weniger einem Nervenschaden geschuldet. Hier nutzt man eine anatomische Besonderheit am Zeigefinger, da hier in der Regel 2 Strecksehnen paarig angelegt sind. Die Strecksehne des Extensor indicis kann dann distal durchtrennt, nach radial verlagert und mit dem distalen Anteil der Extensor-pollicis-longus-Sehne gekoppelt werden.

Nach erfolgter Operation müssen Physiotherapie und Ergotherapie erfolgen. Um die Umlernprozesse zu erleichtern, kann hier neben den klassischen Techniken auch die propriozeptive neuromuskuläre Fazilitationstechnik (PNF) oder Spiegeltherapie angewendet werden. An dieser Stelle sei noch einmal auf die Notwendigkeit der hohen Motivation des Patienten hingewiesen.

\section{Fazit für die Praxis}

- Bei Nervenverletzungen sollte möglichst die Primärversorgung angestrebt werden.

- Ist dies nicht möglich oder erfolgt, ist die sekundäre Nervenrekonstruktion als Option in Betracht zu ziehen. Nach langer Latenz oder im vernarbten Gewebe ist der Erfolg jedoch erheblich reduziert.

- Im Falle von Beeinträchtigungen des Patienten durch motorische Ausfälle sind Ersatzplastiken bei bleibendem oder langfristigem motorischem Ausfall indiziert.

- Wichtige Voraussetzungen sind die Motivation des Patienten und die Gewährleistung von passiv frei beweglichen Gelenken. Dabei ist das Umlernen der neuen Muskelfunktion auch im hohen Alter möglich.
Trauma Berufskrankh 2017 · 19 (Suppl 3):S347-S349

DOI 10.1007/s10039-017-0286-2

๑) Springer Medizin Verlag GmbH 2017

\section{B. Behr}

Sekundäre Wiederherstellung von Nervenverletzungen und motorischer Ersatz an der oberen Extremität

\section{Zusammenfassung}

Verletzungen von großen Nerven an der oberen Extremität können langfristige Einschränkungen für den Patienten bis hin zum Wechsel des Berufs und Berentungen nach sich ziehen. In dem vorliegenden Vortragsbericht werden Voraussetzungen und Strategien zur Rekonstruktion der Verletzungen durch sekundäre Nervenwiederherstellung und motorische Ersatzplastiken vorgestellt.

Schlüsselwörter

Rekonstruktion · Sekundärversorgung ·

Operation · Physiotherapie - Ergotherapie

Secondary reconstruction of damaged nerves and motor replacement in the upper extremities

\section{Abstract}

Injuries to the major nerves in the upper extremities can result in severe disabilities as well as alternative occupations or early retirement for the patient. This talk synopsis focuses on the prerequisites and strategies to reconstruct these injuries by secondary nerve repair as well as tendon transfer.

Keywords

Reconstruction - Secondary treatment

Surgery · Physiotherapy · Ergotherapy

\section{Korrespondenzadresse}

\section{Priv.-Doz. Dr. B. Behr}

Klinik für Plastische Chirurgie und Schwerbrandverletzte, Handchirurgiezentrum, Operatives Referenzzentrum für Gliedmaßentumoren, BG Universitätsklinikum Bergmannsheil Bürkle-de-la-Camp-Platz 1, 44789 Bochum, Deutschland

bjorn.behr@rub.de 


\section{Einhaltung ethischer Richtlinien}

Interessenkonflikt. B. Behr gibt an, dass kein Interessenkonflikt besteht.

Dieser Beitrag beinhaltet keine vom Autor durchgeführten Studien an Menschen oder Tieren.

The supplement containing this article is not sponsored by industry.

\section{Literatur}

1. Altintas AA, Altintas MA, Gazyakan E et al (2009) Long-term results and the disabilities of the arm, shoulder, and hand score analysis after modified Brooks and D'Aubigne tendon transfer for radial nerve palsy. J Hand Surg Am 34:474-478

2. Chuinard RG, Boyes JH,StarkHHetal (1978) Tendon transfers for radial nerve palsy: use of superficialis tendons for digital extension. J Hand Surg Am 3:560-570

3. Friden J, Tirrell TF, Bhola $S$ et al (2015) The mechanical strength of side-to-side tendon repair with mismatched tendon size and shape. J Hand Surg Eur Vol 40:239-245

4. Harhaus L, Hirche C, Giunta RE et al (2017) Strategies on the treatment of nerve injuries accompanied by severe soft tissue damage consensus statement of the German-Speaking Society for Microsurgery of Peripheral Nerves and Vessels. Handchir Mikrochir Plast Chir. doi:10.1055/ s-0043-111408

5. Littler JW (1949) Tendon transfers and arthrodeses in combined median and ulnar nerve paralysis. JBone Joint Surg Am 31 A:225-234

6. Omer GE Jr. (1968) Evaluation and reconstruction of the forearm and hand after acute traumatic peripheral nerve injuries. J Bone Joint Surg Am 50:1454-1478

7. Rosberg HE, Carlsson KS, Hojgard S et al (2005) Injury to the human median and ulnar nerves in the forearm - analysis of costs for treatment and rehabilitation of 69 patients in southern Sweden. JHand Surg Br 30:35-39

8. Shergill G, Bonney G, Munshi P et al (2001) The radial and posterior interosseous nerves. Results fo 260 repairs. J Bone Joint Surg Br 83:646-649

9. Taylor CA, Braza D, Rice JB etal (2008) The incidence of peripheral nerve injury in extremity trauma. Am J Phys Med Rehabil 87:381-385

10. Tsuge K, Adachi N (1969) Tendon transfer for extensor palsy of forearm. Hiroshima J Med Sci 18:219-232 